\title{
Double dissociation between the neural correlates of the general and specific factors of the Life Orientation Test-Revised
}

\author{
Guido Alessandri ${ }^{1}$ • Vilfredo De Pascalis ${ }^{1}$
}

Published online: 9 June 2017

(C) Psychonomic Society, Inc. 2017

\begin{abstract}
In this article, we explore the neural correlates of the general and specific factors assessed by the Life Orientation Test-Revised. These factors have been shown to assess general optimism (GO) and a form of self-enhancement akin to unrealistic optimism (SP). Toward our aim, we used a standardized low-resolution brain electromagnetic tomography (sLORETA), which provides electroencephalographic (EEG) localization measures that are independent of recording reference. Resting-EEG and self-report measures of GO and $\mathrm{SP}$ were collected from 51 female undergraduates. EEGs were recorded across 29 scalp sites. Anterior and posterior source alpha asymmetries of cortical activation were obtained by using the sLORETA method. On the basis of previous research findings, ten frontal and six parietal regions of interest (ROIs) were derived. Alpha asymmetry in the posterior cingulate (i.e., BA31) was uniquely associated with GO. In contrast, SP was associated with areas of the inferior frontal gyrus (BA44, BA45) and with the left subcentralis area (BA43). Theoretical and practical implications of the findings are provided and discussed.
\end{abstract}

Keywords Optimism - Life Orientation Test - Method effect · Method factors $\cdot$ Bifactor model $\cdot$ Inferior frontal cortex

Dispositional optimism is undoubtedly one of the most important psychological constructs to be studied in the last decades. Theoretically, it corresponds to a general orientation toward the future in which one believes that good things will be

Guido Alessandri

guido.alessandri@uniroma1.it

1 Department of Psychology, University of Rome "La Sapienza", Rome, Italy plentiful and bad things scarce (Scheier \& Carver, 1992). A solid literature has shown a positive association between optimism, good physical health, and longevity (Maruta, Colligan, Malinchoc, \& Offord, 2000; Scheier \& Carver, 2001). In contrast, people who are doubtful about their future are more likely to try to push adversities away and to do things that only provide temporary distractions rather than aid in solving their problems (Ben-Zur, Gilbar, \& Lev, 2001). Thus, in an attempt to uncover the dispositional basis of optimism, researchers have begun to explore the neural basis of optimism by investigating the association between electroencephalographic (EEG) hemispheric asymmetry and dispositional optimism (De Pascalis, Cozzuto, Caprara, \& Alessandri, 2013). Examining this association may lead to a better understanding of the specific neural mechanisms underlying optimism (Drevets et al., 1997).

Clearly, for this line of research to be successful, the availability of reliable measures of trait optimism is fundamental. The use of contaminated measures of trait optimism may lead to spurious relations between the constructs and external variables (see Alessandri, Vecchione, Barbaranelli, \& Tisak, 2011). Currently, one of the more popular measure of dispositional optimism is the Life Orientation Test-Revised (LOTR; Scheier, Carver, \& Bridges, 1994). Nonetheless, the dimensionality of the LOT-R is the focus of an ongoing psychometric debate that has potentially wide implications for the measurement of the construct and, thus, for all investigations using the LOT-R to unravel the neural basis of optimism.

In the present study, we aim to further refine our understanding of the neural basis of the optimism trait, as assessed by the LOT-R. We begin by reviewing the psychometric properties of this instrument, highlighting the complexities associated with obtaining reliable individual scores. Then, we introduce and explain a different psychometric representation of the factor structure of the LOT-R. This alternative 
conceptualization, known as a bifactor model, is currently believed to best represent the psychometric structure of this instrument (Rauch, Schweizer, \& Moosbrugger, 2007). This bifactor model includes a general optimism factor, along with a specific factor assessing a particular kind of "selfenhancement" tendency.

The objective of the present study was to use EEG asymmetries in cortical regions of interest to highlight the regional asymmetries associated with the general optimism and specific "self-enhancement" factors obtained by a bifactor model. To this aim, we used the sLORETA method. This research is important for both its methodological development and its significant clinical implications. Indeed, accurately assessing optimism is fundamental to gaining reliable information regarding the neural substrates of optimism. Moreover, clearly localizing optimism is also important in light of its association with an individual's psychological adjustment, and the level of depressive symptoms in particular.

\section{The neural basis of optimism}

In principle, the construct of dispositional optimism is associated with that of approach/withdrawal-related motivational tendencies (De Pascalis et al., 2013). These tendencies reliably relate to frontal alpha asymmetry (FAA), in the form of differences in the relative activation of the frontal cortex (Davidson, 1992; De Pascalis et al. 2013). According to the dispositional model of frontal affective style, based on Gray's (1982) reinforcement theory, increased activity in the left frontal cortical region of the brain enhances the individual's experienced approach motivation (Davidson, 1992; Maxwell \& Davidson, 1992; Sutton \& Davidson, 1997). In contrast, increased activity in the right frontal cortex enhances the individual's experienced withdrawal motivation (Davidson, 1992; Maxwell \& Davidson, 1992; Sutton \& Davidson, 1997). It is important to note that, within this model, the baseline asymmetrical frontal EEG activity reflects a trait (Tomarken, Davidson, Wheeler, \& Kinney, 1992).

Overall, the robustness of a model of frontal affective style has been corroborated in a long series of studies employing measures of asymmetry in brain activity (using EEG). These studies found that both states of behavioral activation (BAS; Amodio, Master, Yee, \& Taylor, 2008; Carver \& White, 1994; Coan \&Allen, 2003; De Pascalis, Variale, \& D'Antuono, 2010; Harmon-Jones \& Allen, 1997; Peterson, Gable, \& Harmon-Jones, 2008; Sutton \& Davidson, 1997) and positive emotional states (Harmon-Jones \& Allen, 1997; HarmonJones \& Sigelman, 2001) are associated with greater left than right frontal brain activity, whereas negative emotional states are associated with greater right than left frontal brain activity.

Previous studies investigating the neural basis of dispositional optimism (and related constructs), have typically followed this general framework. For example, Kingery (2003) reported that tonically increased left-lateralized activity may predispose an individual to information processing biases toward positive cues. De Raedt, Franck, Fannes, and Verstraeten (2008) found a significant positive association between higher left versus right frontal brain activity and selfesteem, a measure related to optimism and approach/ avoidance tendencies. Finally, a large number of studies based on EEG-alpha asymmetry have reported both decreased leftfrontal activation and decreased right-parietal activation associated with depressive symptoms (Davidson, 1985; Henriques \& Davidson, 1991; Schaffer, Davidson, \& Saron, 1983) and experimentally induced depression (van Honk, Schutter, Putman, de Haan, \& d'Alfonso, 2003).

\section{Assessing dispositional optimism: The Life Orientation Test-Revised}

Recently, De Pascalis and colleagues (2013), in reviewing previous studies investigating the neural substrates of optimism, noted the scarcity of studies using direct psychometric measures of optimism for investigating neural substrates (p. 200). Given the relevance of optimism for well-being and adjustment, they suggested that unraveling the association between "traditional and source space derived asymmetry measures ... could provide insight into the mechanisms underlying optimism, and possibly show a way to control its inverse, depression" (p. 200). Currently, the LOT-R represents one of the more promising psychometric scales to be used as a direct measure of dispositional optimism.

The LOT-R was introduced as a brief, one-dimensional, and high face-valid measure of general or dispositional optimism (henceforth, GO; Scheier et al., 1994). The LOT-R represents an improved (and shorter) version of the LOT scale (Scheier \& Carver, 1985) and consists of six items: three positively worded and three negatively worded statements. The popularity of the LOT-R is attested by more than 1,500 citations of the original article in PsycInfo. As assessed by the LOT-R, GO is a broad, generalized and stable (i.e., trait-like) construct, which captures confidence pertaining to life, rather than just to a specific context (Scheier \& Carver, 1992). It is therefore conceptually distinct from specific optimism, which is usually assessed using experimental procedures and is dependent on environmental circumstances (Peterson, 2000).

Although not widely recognized, the dimensionality of the LOT-R is the focus of an ongoing psychometric debate that has potentially wide implications for the measurement of the construct (see Alessandri et al., 2011). Even though the scale was conceived to assess $\mathrm{GO}$ as a single bipolar dimension (i.e., higher scores, higher GO; lower scores, higher pessimism; see Scheier et al., 1994), many factor-analytic studies have suggested that the six items often split into two 
separate dimensions. The first dimension, loaded by the three positive items, was labeled optimism; the second dimension, including the three negative items, was labeled pessimism.

Researchers have interpreted these results in different ways. For example, several authors (Creed, Patton, \& Bartum, 2002; Herzberg, Glaesmer, \& Hoyer 2006; Kubzansky, Kubzansky, \& Maselko, 2004; RobinsonWhelan, Kim, MacCallum, \& Kiecolt-Glaser, 1997) have claimed that optimism and pessimism are potentially independent factors, pointing to their different correlational patterns with several constructs. On closer scrutiny, however, the correlation between the two factors has been found to be inconsistent across samples, and their correlational pattern with external variables is somewhat ambiguous (in this regard, see Rauch et al., 2007).

Consequently, several authors - in primis the scale authors, Scheier and colleagues (1994) - have argued that the observed deviation from unidimensionality is an artifact of item wording and is inconsistent with the theory underlying the LOT-R scale. These authors maintain that the empirical emergence of optimism and pessimism is a clear artifact of item wording, and they have identified a method effect associated with the positively worded items (Meharabian \& Ljunggren, 1997; Scheier et al., 1994; Vautier, Raufaste, \& Cariou, 2003).

Since then, different studies have shown that the method effect factor of the LOT-R has (1) convergent validity across informants (i.e., self- and other-report); (2) discriminant and incremental validity with respect to the GO factor; (3) a strong association with a self-enhancement response style; (4) significant, though not high, heritability (Alessandri et al., 2010; Rauch et al., 2007); and (5) high longitudinal stability (Vecchione, Alessandri, Tisak, \& Caprara, 2014). In sum, this consistent pattern of results suggests that the so-called method effect associated with the positively worded items of the LOT$\mathrm{R}$ meets the criteria defining personality traits.

Alessandri and colleagues (Alessandri et al., 2011; but see also Vecchione et al., 2014) recently offered an overarching interpretation of the two factors assessed by the six items of the LOT-R, one based on the idea that the psychometric structure of the LOT-R resembles a bifactor model (Chen, West, \& Sousa, 2006; Reise, Morizot, \& Hays, 2007). Notably, bifactor models may be considered when (a) a general factor accounts for the commonality of items, (b) there is at least one domainspecific factor, and (c) both the common factor and the domain-specific factors are of interest to researchers (Chen et al., 2006). In the case of the LOT-R, the proposed bifactor model (represented graphically in Fig. 1) includes a general optimism factor (GO) plus a substantive specific factor, tapping a kind of favorability bias in self-description (Paulhus \& John, 1998) that encompasses the tendency to exaggerate one's agentic qualities, such as competence, brightness, intellect, and dominance (Alessandri et al., 2011). This interpretation has been repeatedly put forth by several other authors

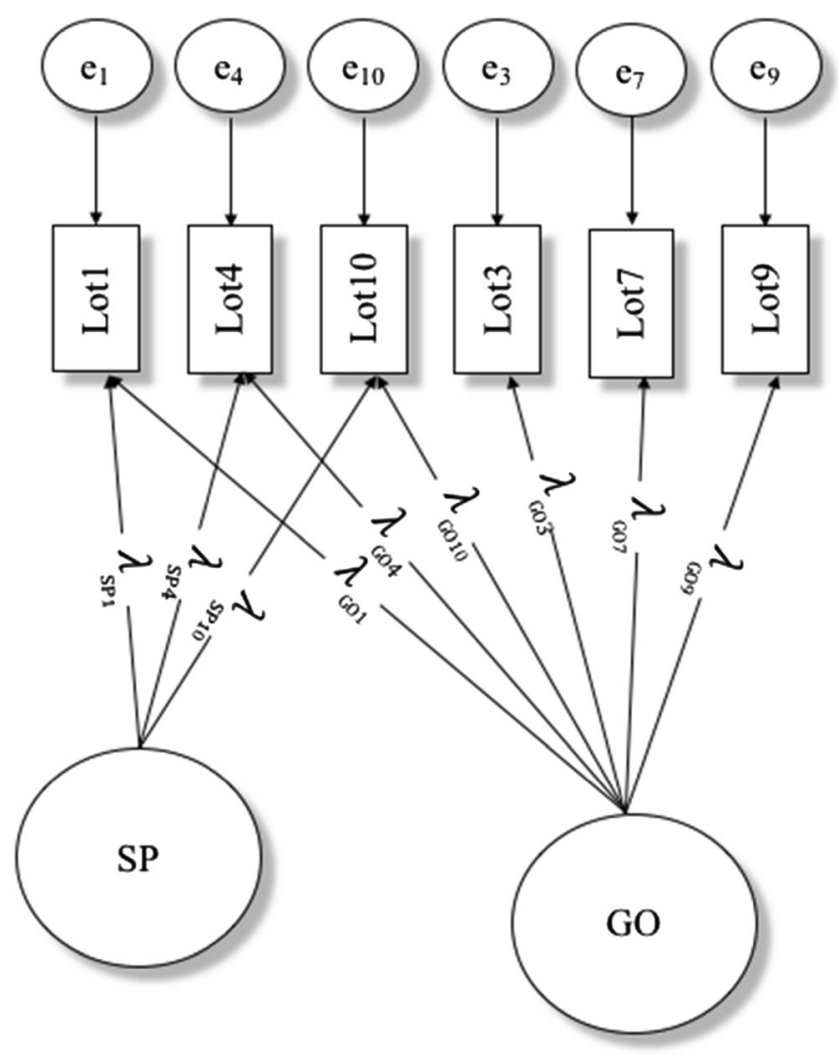

Fig. 1 The proposed bifactor model. Lambdas $(\lambda)$ are indexed by the position of each item in the questionnaire $(1, \ldots, 10)$ and by the factor they belong to; $\mathrm{GO}=$ general optimism; $\mathrm{SP}=$ specific factor; $\varepsilon=$ item residual variances associated with positively $\left(\varepsilon_{1}, \varepsilon_{4}, \varepsilon_{10}\right)$ and negatively $\left(\varepsilon_{3}, \varepsilon_{7}, \varepsilon_{9}\right)$ worded items

(see, e.g., Rauch et al., 2007; Vecchione et al., 2014), who refer to the specific factor of the LOT-R as "selfenhancement" and have found that it is related to selfdeceptive enhancement (Rauch et al., 2007), or a tendency to give favorably biased self-descriptions.

The interpretation of this model can be further refined using the correlated trait-correlated method minus one [CT-C(M-1)] framework (see Eid, Lischetzke, Nussbeck, \& Trierweiler, 2003). Given that a specific factor for the negatively worded items is not considered in the model, it is reasonable to assume that it has been implicitly chosen as the "comparison standard" (i.e., the reference "positive item wording" method). Therefore, the general GO factor captures all the systematic variance that is shared by the three positively worded items and (reversed) by the three negatively worded items. The proportion of the systematic variance shared "only" by the three positively worded items represents the specific (true) factor variance explained by the specific factor, interpreted as the self-enhancement factor (SP).

When observed through the lens of the CT-C(M-1) framework, each factor included in the model may be clearly interpreted. In this regard, previous studies (Alessandri et al., 2011; Rauch et al., 2007; Vecchione et al., 2014) have 
demonstrated that high agreement with positively worded items represents the product of individual's tendency to be unrealistically optimistic about the future. Harris and Hahn (2011) described this tendency as an aspect of a broader selfenhancement bias that encompasses diverse beliefs, such as overly positive illusions about one's own future and abilities (Taylor, Kemeny, Reed, Bower, \& Gruenewald, 2000), overconfidence in judgments involving the self (Kahneman \& Tversky, 1973), and the tendency to overestimate one's own ability to interact with others (Alicke \& Govorun, 2005).

Despite the indications offered by the studies reported above, researchers using the LOT-R routinely rely on summative or mean scale scores of the six items to estimate an individual's optimism score. Unfortunately, scores obtained by this procedure are contaminated (see Alessandri et al., 2011). Indeed, before a bifactor model is applied, the positively worded items from the LOT-R confound (1) variance shared with the general optimism factor, (2) variance shared uniquely with the specific, self-enhancement factor, and (3) measurement error variance. Although the optimism factor usually explains the majority of variance (i.e., about $70 \%$; see Alessandri et al., 2011; Vecchione et al., 2014), the proportion of variance explained by the specific factor is quite substantial, approximately 6\%-7\% (see Alessandri et al., 2011; Vecchione et al., 2014). Thus, caution is necessary when dealing with scores obtained using the LOT-R.

\section{The present research}

The aims of this study were twofold. First, we planned to contribute by refining the empirical search for neural correlates of good optimism, in terms of EEG hemispheric asymmetry, by using standardized low-resolution brain electromagnetic tomography (sLORETA), which improves the localization of EEG activity sources in the brain and provides measures that are independent of the recording reference. In this regard, Kingery (2003) found a positive association between optimism and greater right than left parietal cortical activation. The left prefrontal cortex (PFC) plays a crucial role in the perception, processing, and regulation of emotions, and prefrontal EEG alpha asymmetry reflects a latent trait (Hagemann, Naumann, Thayer, \& Bartussek, 2002). These findings suggest that tonically increased left-lateralized activity may predispose an individual to information-processing biases toward positive cues. De Raedt et al. (2008) showed a significant positive association between higher left-versusright frontal brain activity and a measure of optimism. Functional magnetic resonance imaging (fMRI) studies have also suggested an association between optimism and the activation of anterior regions of the cortex (e.g., Sharot, Riccardi, Raio, \& Phelps, 2007; Somerville, Kelley, \& Heatherton, 2010). Finally, on the basis of EEG-alpha asymmetry observations of both decreased left-frontal activation and decreased right-parietal activation in depressive symptoms (Davidson, 1985; Henriques \& Davidson, 1991; Schaffer et al., 1983), one would expect that both left-frontal and right-parietal activations would be associated with optimism. In this regard, De Pascalis et al. (2013) recently reported an association between higher activation of both the left superior frontal gyrus (BA10) and the right posterior cingulate cortex (BA31) and optimism. We planned to confirm these results, by using a more refined or "purified" measure of optimism.

Second, we aimed to contribute to the psychometrically oriented debate on the nature of the general and specific factors of the LOT-R, by investigating the neural correlates of the SP factor. Previous studies have investigated the neural correlates of a self-enhancement bias. In general, the results consistently suggest that a self-enhancement bias is associated with physiological activity in the left hemisphere (see Hecht, 2013). For example, Tamagni et al. (2010) found that left-ear vestibular stimulation, which momentarily boosts activity in the right hemisphere, made participants significantly less optimistic and more likely to believe that they were vulnerable to health misfortunes in the future. Another study by Sharot and colleagues (Sharot et al., 2012; Sharot et al., 2007) demonstrated that transcranial magnetic stimulation (TMS) interference in the activity of the right inferior frontal gyrus caused healthy participants to be more optimistic. Our aim was to investigate whether higher scores on the specific factor of the LOT-R are positively correlated with indices of higher activation of the left hemisphere and, in particular, with the left inferior frontal gyrus.

\section{Method}

\section{Participants and procedure}

The sample included 51 right-handed, healthy female psychology students (age range between 20 and 34 years; $M=$ $24.1, S D=3.7$ ) who were recruited through advertisements. Since several studies have demonstrated a specific gender difference in oscillatory activity during cognitive tasks (CorsiCabrera, Ramos, Guevara, Arce, \& Gutierrez, 1993; Kamarajan et al., 2008), only women were invited to participate in the study. We excluded individuals with hearing or visual impairment, severe medical conditions that might interfere with vigilance and task performance, neurological and psychiatric illnesses, and drug or alcohol dependence. Participants were rescheduled if they were on their menstrual cycle or were on any medication that could interfere with EEG recording. All participants were required to avoid the use of caffeine, tobacco, or alcohol on the morning of their testing. Before beginning the EEG recordings, participants signed informed consent forms. Experimental procedures and ethical 
guidelines were in accordance with approval from the Sapienza, University of Rome ethics committee. At the beginning of the session, each participant was seated in an electrically shielded EEG booth, and electrodes were applied to measure electrooculography (EOG) and EEG. Eight 1-min EEG intervals were recorded. Participants were instructed to keep their eyes open for four of the 1-min intervals and closed for the other four 1-min intervals, in an order that was counterbalanced across participants. Participants were instructed to remain quiet and to inhibit blinks or eye movements during each recording period. During the eyes-open condition, participants had to fixate on a white cross displayed in the center of a computer monitor that was $1.2 \mathrm{~m}$ distant from their head. The resting-EEG data were derived from a previous study on EEG-alpha asymmetry and personality published elsewhere (De Pascalis et al., 2013).

\section{Instrument: The Life Orientation Test-Revised}

The Life Orientation Test-Revised (LOT-R; Scheier et al., 1994) is composed of ten items, of which four are "filler" or control items, and thus are regularly excluded from the total composite score. Of the remaining six items, three (i.e., Item 1: "In uncertain times, I usually expect the best"; Item 4: "I'm always optimistic about my future"; and Item 10: "Overall, I expect more good things to happen to me than bad") are positively worded, and three are negatively worded (i.e., Item 3: "If something can go wrong for me, it will"; Item 7: "I hardly ever expect things to go my way"; and Item 9: "I rarely count on good things happening to me"). Participants report their degree of agreement with each statement on a fivepoint scale. The responses to negatively worded items are routinely reverse-scored, so that larger composite scores (i.e., scores computed as the sum of the individual scores on all items) indicate higher levels of optimism. However, given the bifactorial structure of the LOT-R, this approach is not reliable. Instead, researchers have recommended the use of weighted individual scores, such as factor scores derived from a confirmative (bi)factor analysis (see Alessandri et al., 2011). These factor scores are naturally purified by the simultaneous influences of the GO and SP factors (plus measurement error) on the three positively worded items. Indeed, each individual concurrently obtains (1) a score on the GO dimension and (2) a score on the SP dimension, which are theoretically independent. We describe below the procedures that we followed to fit a bifactor model to our data and how the factor scores on the GO and the SP factors were obtained. Finally, although the use of the Cronbach alpha coefficient is not entirely appropriate with scales that deviate from unidimensionality (Sijtsma, 2009), we present values of this coefficient for the sake of comparability with previous studies. In general, Cronbach's alpha attested to the very good internal reliability (i.e., .80) of the LOT-R in the present sample. The average individual score on the six items was $17.11(S D=3.49)$.

\section{EEG recording}

Scalp EEG was recorded from 13 lateral pairs of electrodes (Fp1, Fp2, F7, F8, F3, F4, FT7, FT8, T3, T4, FC3, FC4, C3, $\mathrm{C} 4, \mathrm{CP} 3, \mathrm{CP} 4, \mathrm{TP} 7, \mathrm{TP} 8, \mathrm{~T} 5, \mathrm{~T} 6, \mathrm{P} 3, \mathrm{P} 4, \mathrm{O} 1, \mathrm{O} 2, \mathrm{~A} 1$, and $\mathrm{A} 2)$ and from five midline electrodes $(\mathrm{Fz}, \mathrm{FCz}, \mathrm{CPz}, \mathrm{Pz}$, and $\mathrm{Oz}$ ) using a pure-tin electrode electrocap (Electro Cap International, Inc.; Blom \& Anneveldt, 1982). The Cz electrode was used as a reference, and a ground electrode was located on the forehead. Bipolar vertical and horizontal EOGs were recorded, respectively from supra- and infraorbital positions around the right eye and from the epicanthi of the right and left eyes by tin electrodes. The electrodes' impedances were below $5 \mathrm{k} \Omega$. EEG was recorded through a 40-channel NuAmp acquisition system (Neuroscan Inc., Herdon, VA, USA) set in DC mode with a gain of 200 (100 for the EOG channels). The signals were sampled at $1024 \mathrm{~Hz}$ and band-passed from 0.01 to $48 \mathrm{~Hz}$ using a Butterworth zerophase filter with 24-dB/octave roll-off. The EEG data analysis was conducted offline using the Brain Vision Analyser software (v2.0; Brain Products) for preprocessing and implementation of the eye movement correction procedure. Each combined EEG and EOG record was visually inspected offline for artifacts. The resting eyes-open and eyes-closed EEG signals were segmented into 2 -s epochs. Channels were then automatically marked bad when they exceeded a transition threshold of $150 \mu \mathrm{v}$ over the entire segment (max-min). Eye movements were detected when the horizontal eye channels exceeded a threshold of $100 \mu \mathrm{v}$ (max-min) over a 200-ms time window. All segments were visually inspected by a trained research assistant. Each portion of the EEG recordings that showed ocular, muscular, or movement artifacts on any channel was rejected for this and all simultaneously recorded channels (Barlow, 1986; Hagemann, Naumann, \& Thayer, 2001). After artifact rejections, an ocular correction procedure was performed on the segmented EEG data in accordance with Gratton, Coles, and Donchin's (1983) method.

\section{SLORETA cortical source analysis of EEG}

The sLORETA (Pascual-Marqui, 2002) method was used to estimate the intracerebral electrical current density sources underlying alpha $(8-13 \mathrm{~Hz})$ activity recorded at the scalp by using a forced-average reference. sLORETA is a linear, inverse algorithm that enables the spatial identification and analysis of brain cortical activity via conventional EEG recordings (Fuchs, Kastner, Wagner, Hawes, \& Ebersole, 2002; Jurcak, Tsuzuki, \& Dan, 2007; Pascual-Marqui, 2002). sLORETA computes current density (i.e., the amount of electrical current flowing through a solid) without assuming any number of 
active sources. The solution space of sLORETA is restricted to cortical and some hippocampal and amygdala gray matter, defined via a reference brain from the Brain Imaging Center at the Montreal Neurological Institute (MNI: Collins, Neelin, Peters, \& Evans, 1994; Mazziotta et al., 2001). The sLORETA implementation incorporates a 3-D-shell spherical head model registered to a recognized anatomical brain atlas (Talairach \& Tournoux, 1988). sLORETA performs source localization in 6,239 cortical gray matter voxels of $5 \mathrm{~mm}^{3}$ size; localization inference is based on standardized values of the current density estimates (Wagner, Fuchs, \& Kastner, 2004).

In the present study, the EEG was first reconstructed into nonoverlapping epochs of 2,048 ms. After artifact detection, all available artifact-free EEG epochs (on average, 177.5 epochs across participants, $S D=31.1$; about 6 min of EEG recording) were extracted from both the eyes-closed and eyesopen trials. The EEG signal was then resampled at $256 \mathrm{~Hz}$, and using the sLORETA software we computed the overall average cross-spectrum, calculated across both the eyes-open and eyes-closed EEG epochs for each participant. These cross-spectral matrices served as the input for the sLORETA source analysis to calculate the sources of current density. sLORETA was settled to yield alpha power $(8-13 \mathrm{~Hz})$ of the current density at each voxel. Alpha source activities were obtained from many regions of interest (ROIs): Brodmann areas (BAs) 1, 2, 3, 4, 5, 6, 7, 8, 9, 10, 11, 23, 24, 25, 31, $32,33,34,39,40,43,44,45,46$, and 47 (see De Pascalis et al., 2013). The mean alpha power of each ROI was calculated over all voxels belonging to the same anatomical structure. To reduce skew, the global power values for each ROI at each site were submitted to a natural $\log$ transformation and averaged across all artifact-free epochs. For each left- and right-hemisphere ROI, an alpha asymmetry score was calculated as the right log-alpha power minus the left log-alpha power. Because alpha power is inversely related to cortical activity (Lindsley \& Wicke, 1974), higher scores indicate greater left-sided cortical activity.

\section{Statistical analysis}

Deriving individual scores for GO and SP To derive individuals' scores on the GO and SP factors, we fit the bifactor model including the GO and SP factors described above by using Bayesian structural equation modeling (BSEM), as implemented in Mplus 7.11 (Muthén \& Muthén, 2012). BSEM has advantages when used with small sample sizes (Scheines, Hoijtink, \& Boomsma, 1998; Song \& Lee, 2012), particularly when informative priors are used (McNeish, 2016). Since BSEM does not rely on a large-sample normal theory as the maximum likelihood approach does, it accommodates skewed distributions of parameter estimates better and shows better small-sample performance.
In Bayesian inference, the parameters are viewed as variables, and the term "prior" is used to refer to the parameter distributions (Yuan \& MacKinnon, 2009). Depending on the information available, priors can be informative or diffuse (i.e., noninformative). One way to obtain information about a prior in SEM is though summaries of previous studies testing the same model, or by a meta-analysis. Thus, we built our expectations, or "priors," with regard to the model parameters by conducting a quantitative meta-analysis of previous studies that had tested the bifactor model of the LOT-R. This seemed a reasonable approach to derive empirically based expectations to be used as informative priors (Depaoli \& van de Schoot, 2017) for the bifactor model parameters. When an informative prior is specified in the first step of a Bayesian estimation, the prior distributions for the parameters represent the current knowledge, in the form of an expectation as to the likely values of the parameters.

After a prior has been specified through Bayes's theorem, the data can be used to provide information about a parameter value and modify the prior into a posterior that gives the Bayesian estimate (Muthén \& Asparouhov, 2012). The posterior is a compromise between the prior and the likelihood, and can be thought of as the distribution of the data given the parameter value. Broadly speaking, the posterior distribution represents the updated researcher's belief after incorporating the experimental data.

Individual Bayesian plausible values (factor scores) for GO and SP were obtained by taking the mean of the plausible values through multiple imputations for missing values corresponding to the latent variables of GO and SP (Muthén \& Muthén, 2012). Bayesian plausible values are more accurate than maximum likelihood factor scores with small sample sizes, and they produce more accurate results in secondary analyses, such as, for example, when computing correlations between factors (Muthén \& Muthén, 2012).

Model evaluation Model estimation was performed with a default of 10,000 iterations, as compared to the 50,000 iterations typically performed for models using the Markov chain Monte Carlo algorithm and the Gibbs sampler (Muthén \& Asparouhov, 2012). Model convergence was assessed with the potential scale reduction factor (PSRF) diagnostic (Gelman, Carlin, Stern, \& Rubin, 2004). A PSRF value of 1.1 or smaller was used as evidence of convergence. BSEM model fit was assessed with the posterior predictive $p$ value and the associated $95 \%$ credibility interval (Muthén \& Asparouhov, 2012). Credibility intervals represent the Bayesian analogous of frequentist confidence intervals, from which they differ in computational and philosophical respects (but see Gelman et al., 2004). A low posterior predictive $p$ value $(<.05)$ and a positive $95 \%$ lower interval limit indicate a poor model fit, and a good model fit is expected to show a 
posterior predictive $p$ value of approximately .5 and a symmetric $95 \%$ credibility interval (CI95\%) centered around zero.

Neural correlates of GO and SP Zero-order correlations were used for examining the predictive values of the two individual scores for GO and SP with respect to alpha asymmetry scores for each ROI. These correlations were computed in Mplus by using a noninformative prior and an inverse Wishart distribution: $I W(.00,-12)$. The significance of correlations and $t$ tests was assessed by (1) looking at the $95 \%$ credibility interval and (2) conducting a default Bayesian hypothesis test for the presence of a correlation $(B-P C$; Wetzels \& Wagenmakers, 2012).

Credibility intervals are informative regarding the plausible values eventually assumed by a parameter. The $B-P C$ calculation uses the Bayes factor to compute the probability of the observed data under the null hypothesis (i.e., that the correlation is equal to 0 ) with respect to the alternative hypothesis (i.e., that the correlation is not equal to 0 ), and is robust to multiple comparisons. Thus, it is able to quantify the degree of evidence in support of the alternative hypothesis.

When the Bayes factor has a value greater than 1 , this indicates that the data are more likely to have occurred under the alternative hypothesis (H1) than under the null hypothesis (H0), and vice versa when the Bayes factor is less than 1 . Jeffreys (1961) suggested that Bayes factor values of 1 represent substantially "no evidence" in favor of H1 (i.e., that $r$ is not equal to 0 ); values in the range of 1-3 provide "anecdotal" evidence for $\mathrm{H} 1$; values in the range of 3-10 provide "substantial" evidence for $\mathrm{H} 1$; and values greater than 10 provide "strong" evidence for H1. We considered significant (i.e., $\neq 0$ ) those correlations resulting in both a CI95\% that did not include zero and a $B-P C$ greater than 4 (Jeffreys, 1961).

\section{Results}

\section{Results from the meta-analysis of previous studies}

Research articles reporting tests of the bifactor structure of the LOT-R were found primarily by searching the PsycInfo databases using the keywords optimism, life orientation test revised, LOT-R, method factor, specific factor, and each possible combination of these terms. We then reviewed the reference list of each of these articles to locate additional articles. Only articles that tested the bifactor model and reported enough information about each parameter of interest (i.e., factor loadings associated with each item, item residual variances, and variances of both the GO and SP factors) were included.

The statistic of interest for the present study was the weighted average parameter (i.e., the factor loadings, or $\lambda$, and item residuals, or $\varepsilon$ ), which provides an estimate of the average size of the parameter across different samples, weighted by the number of cases involved in each sample. The standardized loadings were $r$-to-z transformed before computing the averages, and the resulting weighted values were then $z$-to$r$ back-transformed. To obtain an estimate of the across-study variation of the parameter values, we computed the standard deviation weighted for sample size.

Using the search criteria specified above, we identified five published articles reporting information useful for our purposes (i.e., Alessandri et al., 2011; Alessandri et al., 2010; Rauch et al., 2007; Vautier et al., 2003; Vecchione et al., 2014). The total sample size was 13,374 . One study contained two studies conducted on two different samples. In the presence of a longitudinal model (as in Vecchione et al., 2014, Study 2), we estimated the average parameter value across waves for each parameter of interest and then used this value as the study value for further aggregation.

The main findings from this meta-analysis are presented in Table 1 and can be used to outline values for the informative priors necessary to build our Bayesian bifactor model. The values of these priors were the meta-analytic estimates of the averaged values of loadings, item variances, and their variabilities, estimated across the previously published studies. In particular, the weighted mean and variance for each loading were used as informative priors, using a normal distribution. The weighted means and variances for the item residual variances were used as informative priors, but using an inverseWishart distribution (see Muthén \& Asparouhov, 2012). The covariance between GO and SP was fixed to 0 , as in previous studies. Standardized variables were analyzed, and the factor variances were fixed to 1 in order for the priors to correspond to the standardized loadings. This model (21 parameters estimated) converged properly $(\mathrm{PSRF}=1.038)$ and displayed a reasonable fit, with a posterior predictive $p$ value of .18 and a symmetric $95 \%$ posterior predictive interval ranging from 0.909 to 27.86. Posterior parameter estimates, along with credible intervals, are reported at the bottom of Table 2. As can be seen, all estimated parameters were significantly different from zero.

\section{Neural correlates of GO and SP}

The zero-order correlations between individuals' estimated factor scores on GO and the ROIs, along with their 95\% credibility intervals, are presented in Fig. 2. As can be seen, we found substantial evidence regarding an association between higher activation (i.e., a higher increase of alpha activity in the left relative to the right hemisphere) in the right-posterior cingulate cortex (BA31) and GO (similar to that reported by De Pascalis et al., 2013). This result was confirmed both by the credibility interval and a B-PC of 4 . The association between $\mathrm{GO}$ and higher activation of the left superior frontal gyrus (BA10) was not confirmed. With regard to SP, inspection of 
the $95 \%$ credibility intervals suggested significant associations with increased activation in three regions of the left inferior frontal gyrus (IFG): the pars opercularis (BA44), the pars triangularis (BA45), and the pars orbitalis (BA47). Likewise, significant associations were found with higher activation in the left BA46 region, which roughly corresponds to the dorsolateral prefrontal cortex (DLPFC), and increased activation in the left area subcentralis (BA43). According to the B-PC, evidence supporting the inference that the observed $r$ values were higher than 0 was very strong for BA44 $(\mathrm{B}-\mathrm{PC}=$ 44) and BA45 (B-PC = 44); was nearly substantial for BA43 $(\mathrm{B}-\mathrm{PC}=4)$; and was anecdotal for BA46 $(\mathrm{B}-\mathrm{PC}=2)$ and BA47 (B-PC $=1)$.

\section{Sources of activation}

To further understand individual differences the left and right hemispheres for the ROIs, we ran an additional set of analyses. First, we divided participants into those with high and low GO and SP, using a median split. Then we examined the difference in the EEG-alpha current source estimates in each left-right ROI for participants with high and low scores on the constructs. An increased raw EEG alpha current source estimate means a temporary decrease in activation (i.e., an inhibition) in the same ROI. The existence of significant mean differences in levels of activation was investigated by using the automatic Jeffreys-Zellner-Siow (JZS) Bayesian $t$ test that was proposed by Rouder, Speckman, Sun, Morey, and Iverson (2009). The JZS test is able to quantify support in favor of the null $\left(\mathrm{B}-\mathrm{H}_{01}\right)$ or the alternative $\left(\mathrm{B}-\mathrm{H}_{10}\right)$ hypothesis. This information comes in the form of a scaled JZS Bayes factor (BF), the values of which are interpreted as a correlation (see above).

The results are presented in Fig. 3. In particular, strong evidence of inhibition (i.e., higher alpha power) of the right posterior cingulate (BA31; panel a) was found for participants below the median $\mathrm{GO}$ factor score. With regard to SP, we found substantial evidence of higher inhibition of both the left area subcentralis (BA43) and left pars triangularis (BA45), but anecdotal evidence of higher inhibition of the left pars opercularis (BA44). ${ }^{1}$

\footnotetext{
${ }^{1}$ For the sake of comparison with previous research, we also computed the association between individuals' mean scores on all six items composing the LOT-R and all ROIs. In line with previous studies (De Pascalis et al. 2013), the results attested to an association between higher activation in the right posterior cingulate cortex (BA31) and individuals' mean scores on the LOT-R scale. No other significant associations with any other ROI were detected.
}

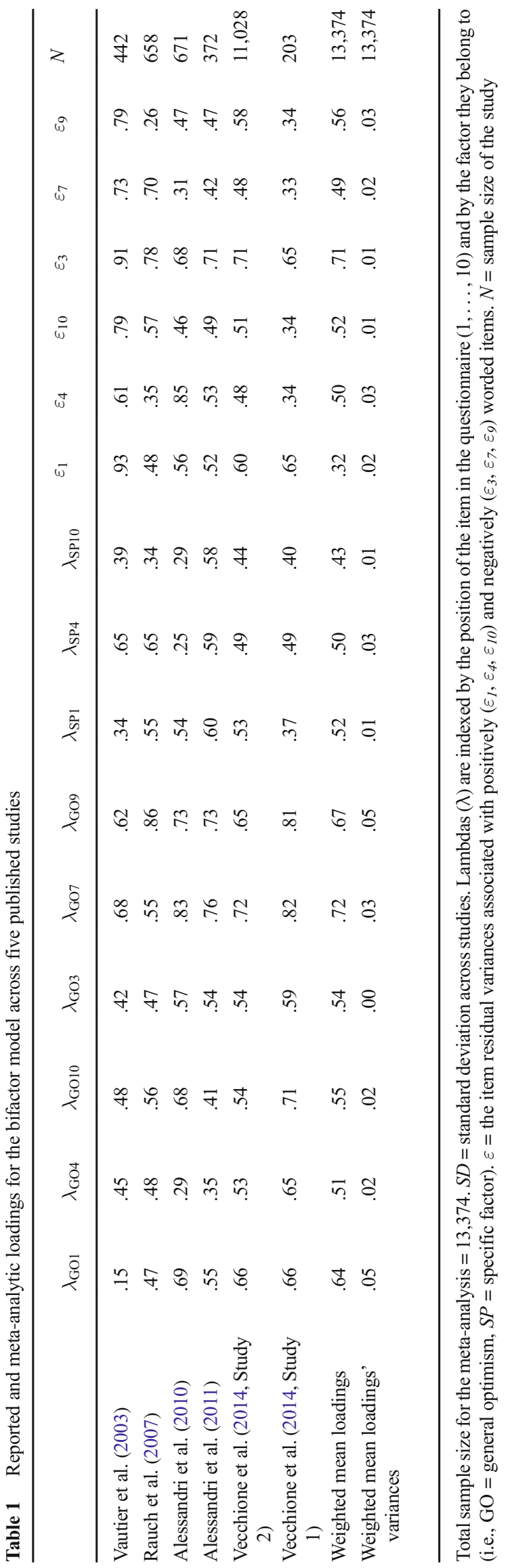




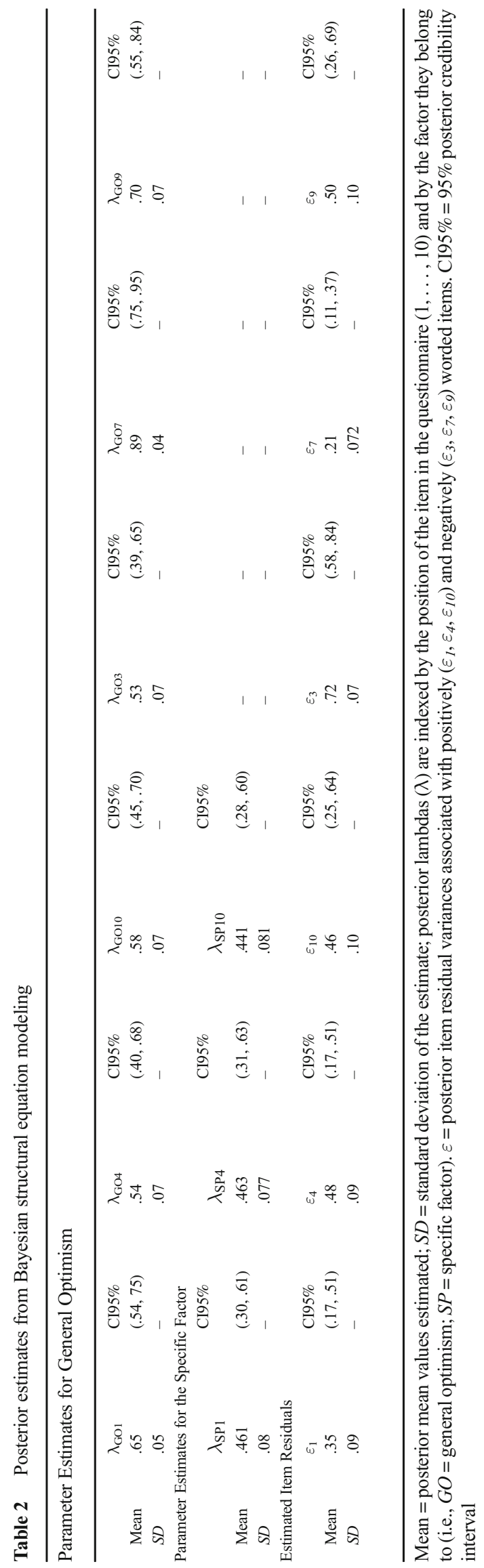

\section{Discussion}

The Revised Life Orientation Test is one of the most popular and widely used instruments to assess dispositional optimism (Scheier et al., 1994). The popularity of the six-item scale is due in part to its long history, its uncomplicated language, and its brevity (it takes only 1 or 2 min to complete). Despite its privileged place in the literature, the bifactorial nature of the instrument has only recently been firmly established, and the external validity of the GO and SP factors deserves further investigation. The contribution of our findings is twofold. First, our data add to clarification of the neural correlates of the GO and SP factors. Second, the present results are intended to strengthen our understanding of the ontological status of the GO and SP factors, by providing clues about their specific associations with differential activation in key brain areas.

\section{What is the methodological contribution of this study?}

From a psychometric point of view, our findings corroborate the idea that the routine use of a method factor to aid the fit of a factor model can unwittingly lead researchers to underestimate the potential existence of a substantive bifactor structure. This problem is likely due to the relatively shallow penetration of bifactor models in the field of personality and individual differences, in comparison to the research literature on intelligence (Reise et al., 2007). This may be a result of the increased cost, in terms of theoretical sophistication, required by these models, as well as of the belief that a bifactor model is often less analytically stable than a general factor model.

With regard to the complexity of bifactor models, we note that as in the case of the LOT-R, a specific factor often has the potential to simplify a highly complicated theoretical debate-for example, by supporting an unmet theoretical exigency of empirical unidimensionality (see Alessandri et al., 2011, for a discussion). With respect to the alleged instability of bifactor models, we note that the stability of a bifactor structure is often not lower than that of more diffuse secondorder models (Chen et al., 2006). In our experience with the LOT-R, convergence difficulties while fitting the bifactor model across many different samples has never been an issue.

\section{Are GO and SP associated with the activation of different brain areas?}

We found a series of different neural correlates for GO and SP. In particular, our results refined findings from De Pascalis et al. (2013) and suggested that higher GO is related to a right-sided cortical activation at the posterior cingulate (BA31), but not at the superior frontal gyrus (BA10). The finding that higher optimism is related to enhanced activity in the right posterior cingulate is consistent with Kingery's 


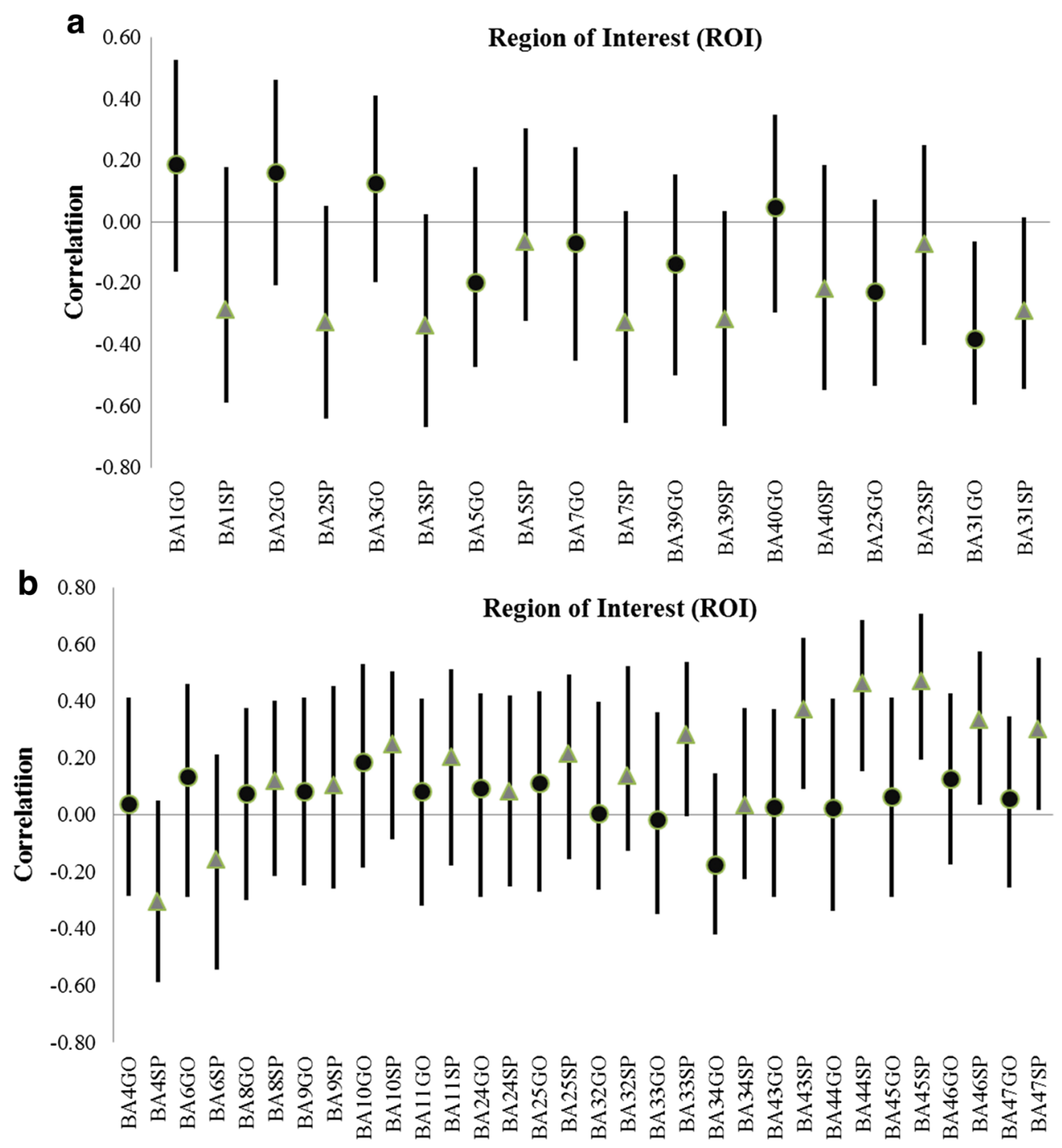

Fig. 2 Zero-order correlations (with credibility intervals) of the general optimism (GO) and specific (SP) factors with parietal (A) and frontal (B) regions of interest

(2003) earlier report of a significant association between optimism and EEG activation in the right parietal cortex. Moreover, this finding is important to appropriately plan future studies aimed to uncover a fine-grained localization of the cortical structures associated with GO.

The most novel finding of this study regards the distinctive and robust associations of SP with the activation of specific areas of the frontal cortex in the left hemisphere. In particular, we found very strong support for the association of SP with left-hemisphere asymmetry at both BA44 and BA45. These cytoarchitectonic areas, together with BA47, are part of the inferior frontal gyrus (IFG). Researchers agree that the right IFG - in particular, areas 44 and 45 (which were found to be relatively inhibited in participants with higher scores on SP) is involved in executive control (Aron, Robbins, \& Poldrack, 2014) or the ability to "schedule and optimize subsidiary processes implemented by posterior cortical and subcortical regions" (Aron et al., 2014, p. 170; Miller \& Cohen, 2001).
The IFG is among the latest brain regions to develop during both ontogenesis and phylogeny (Nakamura et al., 2002). Structurally, it is among the most widely connected regions of the PFC. Indeed, it receives polymodal inputs from many posterior cortical areas and has an intricate network of connections with neighbor areas of the PFC. It has been argued that the IFG exerts executive control by selective suppression of inappropriate responses, such as canceling an unintended movement during go-no-go trials (Rubia et al., 2005), switching from one task to another (Aron, Monsell, Sahakian, \& Robbins, 2004), or blocking unwanted, interfering memories (Anderson et al., 2004).

The present study demonstrated a substantial association between SP and higher activation of left-hemisphere BA43 and some evidence for an association between SP and higher activation of the left-hemisphere BA46. BA43 (which is inhibited in participants with low scores on SP) is positioned in the postcentral gyrus, between the ventrolateral extreme of 

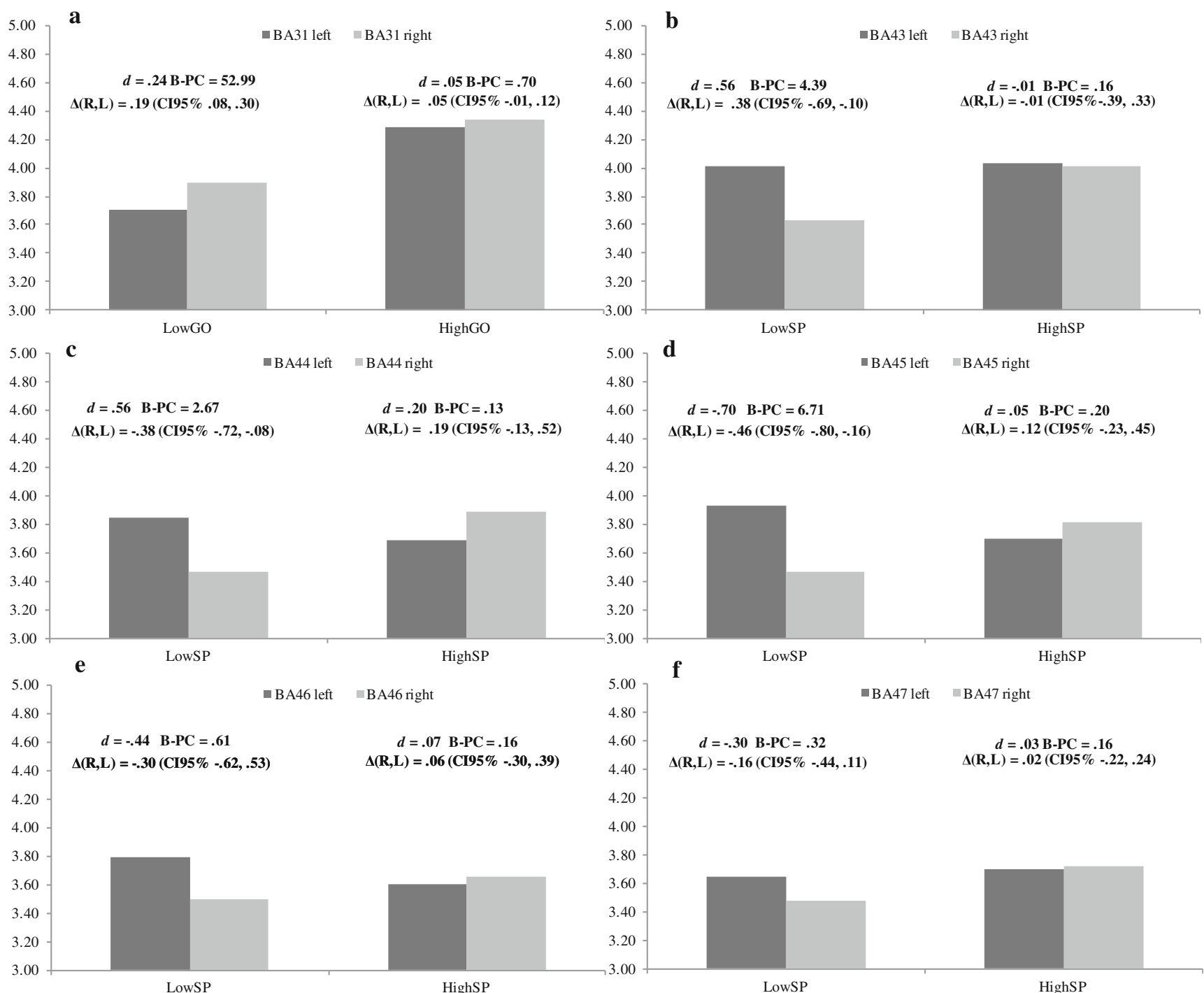

Fig. 3 Relationships between individual factor scores on general optimism (GO) and the specific factor (SP) and raw EEG alpha current source estimates in each region of interest (ROI). B-PC, Bayesian hypothesis test for correlation (values greater than 4 indicate significant evidence

for the alternative hypothesis); $\Delta$, raw differences between logtransformed global power values for the left and right ROIs. Values on the $y$-axis are current density values (i.e., $\mu \mathrm{A} / \mathrm{mm}^{2}$ )

the central sulcus and the lateral sulcus, in the insula. Activation of left BA43 has often been associated with the processing of semantic linguistic stimuli in working memory. Thus, this inhibition might signal difficulties with semantic processing. The right BA46 area is, instead, an area classically associated with decision making in general (Krain et al., 2006) and, in particular, with ambiguous decision making (ADM; Fectau et al., 2007). ADM arises during tasks that require a selection among responses of equal valence and do not involve any apparent risk for the participant (Zelazo \& Müller, 2002). Theoretically, ADM is considered the expression of a "cool" executive function, which has been repeatedly associated with activation at right BA46 during tasks requiring participants to make a choice (Krain et al., 2006). However, the source of the alpha asymmetry was unclear, and the differential activation/inhibition of the right versus the left hemisphere was hard to prove. Thus, these results need to be replicated in future studies.

All in all, the pattern of associations above is in accordance with the current theoretical interpretation of the SP factor, which is conceptualized as an individual stable tendency linked to emotional adjustment and a self-enhancement bias (Alessandri et al., 2011). Some researchers have noted that individuals tend to hold positive illusions (Taylor et al., 2000). In a certain sense, these positive illusions represent a source of resilience that individuals are likely to use in coping with environmental adversities. This is especially true for narcissistic individuals, who hold unrealistically exaggerated beliefs about their abilities and achievements (John \& Robins, 1994). Following this line of reasoning, this specific factor reflects the existence of a secondary trait, assessed by the 
LOT-R contemporaneously with the GO factor, representing an individual's tendency to hold positive beliefs about his or her own future. Thus, participants with higher scores on the SP factor seem to have a stable and generalized tendency to see themselves as unrealistically positive, as can be inferred from the content of the positively worded items of the LOT-R.

It is important to note that the SP factor is statistically and conceptually unrelated to GO. This independence can be maintained only when using a bifactor model to estimate individuals' scores on these factors. Researchers should be aware that using the overall mean score, instead, would result in a polluted measure. Thus, to obtain reliable scientific information regarding the brain structures associated with GO, researchers should consider both factors in practical applications. Understanding the nature of the SP factor and the brain structures with which it is associated may contribute to furthering our understanding of the nature of self-enhancement and of its differences and similarities with GO.

From a more psychometrically oriented point of view, our data offer important indications regarding the way in which individuals high in SP process linguistic stimuli. Indeed, the activation of the left IFG in participants with lower scores on SP may likely signal an increased ability to realistically evaluate the content of the positively worded LOT-R items. These items, as was noted by previous authors (McPherson \& Mohr, 2005), are related to positive and somewhat unrealistic expectations (e.g., "I'm always optimistic about my future"). It is likely that low-SP participants invest more effort in elaborating their response to these items, and this ultimately leads them to offer an answer that is less biased toward unrealistic optimism. This interpretation might be further exacerbated by the contemporaneous higher activation of left BA43 and BA46. Reduced functioning of the first area is likely to compromise the ability of individuals to deeply process the semantic content of the items. The reduced functioning of the second area, instead, may make individuals more acquiescent when responding to positively worded items (i.e., they may express agreement with the item's content) because of a reduced sensitivity to the ambiguity of the items' formulation.

\section{What are the practical implications of the bifactor model?}

We believe our research is significantly relevant for the field of dispositional optimism research, which has classically relied on the LOT-R scale. In the context of theory building, for example, researchers need to be aware that the scale does not measure a single, general factor. It should be noted that the present results do not necessarily question the practical usefulness of the LOT-R in applied contexts, nor calls into question its usefulness for screening purposes given that the GO factor explains a significant portion of items' variance. However, we surmise that using a total score as a reliable proxy of individual GO may be suboptimal, as this total score could be contaminated by different sources of variance. Recognizing the different variance components tapped by the LOT-R items may give researchers additional useful information.

In addition to these practical and theoretical benefits, other conceptual advances may follow from carefully considering our bifactorial model. For instance, theories regarding the nature and adaptive function of key brain areas might benefit from simultaneously investigating the individual correlates of GO and SP, which seem to predict very different patterns of brain activation. Likewise, theories in clinical neuropsychology could try to link each of the two latent factors of the LOT-R to the functioning of the brain in the same clinical phenomena (i.e., depression, anxiety, etc.), to better understand the relationship between different facets of generalized positive expectations.

\section{Conclusion}

Even though several studies have supported a bifactor model as the best psychometric representation of the LOT-R items, the potential theoretical and practical consequences of this model are still far from being widely understood. We hope to have provided new data supporting the view that the LOT-R is best represented by a bifactor model (Chen et al., 2006). Each factor (i.e., GO and SP) is linked with specific brain correlates that should be confirmed in future studies. We would emphasize that the present findings do not necessarily reduce the value of previous studies based on the LOT-R total score. On the basis of this study, it is difficult to know the extent to which the use of a more appropriate structure for the LOT-R would change the results of studies using a onefactor model. However, one should bear in mind that the LOT$\mathrm{R}$ measures two distinct aspects of generalized outcome expectancies. Focusing on one, or on both, is a researcher's choice. Neglecting one factor and using the average score, however, is a flawed procedure.

\section{References}

Alessandri, G., Vecchione, M., Barbaranelli, C., \& Tisak, J. (2011). Investigating the nature of method factors through multiple informants: Evidence for a specific factor? Multivariate Behavioral Research, 46, 625-642. doi:10.1080/00273171.2011.589272

Alessandri, G., Vecchione, M., Fagnani, C., Bentler, P. M., Barbaranelli, C., Medda, E., ... \& Caprara, G. V. (2010). Much more than model fitting? Evidence for the heritability of method effect associated with positively worded items of the revised Life Orientation Test. Structural Equation Modeling, 17, 642-653. doi:10.1080/ 10705511.2010.510064 
Alicke, M. D., \& Govorun, O. (2005). The better-than-average effect. In M. D. Alicke, D. A. Dunning, \& J. I. Krueger (Eds.), The self in social judgment (pp. 85-106). New York: Psychology Press.

Amodio, D. M., Master, S. L., Yee, C. M., \& Taylor, S. E. (2008). Neurocognitive components of the behavioral inhibition and activation systems: Implications for theories of self-regulation. Psychophysiology, 45, 11-19. doi:10.1111/j.1469-8986.2007. 00609.x

Anderson, M. C., Ochsner, K. N., Kuhl, B., Cooper, J., Robertson, E., Gabrieli, S. W., ... \& Gabrieli, J. D. E. (2004). Neural systems underlying the suppression of unwanted memories. Science, 303, 232-235. doi:10.1126/science.1089504

Aron, A. R., Monsell, S., Sahakian, B. J., \& Robbins, T. W. (2004). A componential analysis of task-switching deficits associated with lesions of left and right frontal cortex. Brain, 127, 1561-1573. doi:10. 1093/brain/awh169

Aron, A. R., Robbins, T. W., \& Poldrack, R. A. (2014). Inhibition and the right inferior frontal cortex. Trends in Cognitive Sciences, 8, 170 177. doi:10.1016/j.tics.2004.02.010

Barlow, J. S. (1986). Artifact processing (rejection and minimization) in EEG data processing. In F. H. Lopes da Silva, W. Storm van Leeuwen, \& A. Rémond (Eds.), Handbook of electroencephalography and clinical neurophysiology: Vol. 2. Clinical applications of computer analysis of EEG and other neurophysiological signals (Rev. ed., pp. 15-62). Amsterdam, The Netherlands: Elsevier.

Ben-Zur, H., Gilbar, O., \& Lev, S. (2001). Coping with breast cancer: Patient, spouse, and dyad models. Psychosomatic Medicine, 63, 3239. doi: $10.1037 / 0278-6133.20 .1 .20$

Blom, J. L., \& Anneveldt, M. (1982). An electrode cap tested. Electroencephalography and Clinical Neurophysiology, 54, 591594. doi:10.1016/0013-4694(82)90046-3

Carver, C. S., \& White, T. L. (1994). Behavioral inhibition, behavioral activation, and affective responses to impending reward and punishment: The BIS/BAS Scales. Journal of Personality and Social Psychology, 67, 319-333. doi:10.1037/0022-3514.67.2.319

Chen, F. F., West, S. G., \& Sousa, K. H. (2006). A comparison of bifactor and second-order models of quality of life. Multivariate Behavioral Research, 41, 189-225. doi:10.1207/s15327906mbr4102_5

Coan, J., \& Allen, J. (2003). The state and trait nature of frontal EEG asymmetry in emotion. In K. Hugdahl \& R. J. Davidson (Eds.), The asymmetrical brain (pp. 565-615). Cambridge: MIT Press.

Collins, D. L., Neelin, P., Peters, T. M., \& Evans, A. C. (1994). Automatic 3D intersubject registration of MR volumetric data in standardized Talairach space. Journal of Computer Assisted Tomography, 18, 198-205. doi:10.1016/0022-3956(75)90026-6

Corsi-Cabrera, M., Ramos, J., Guevara, M. A., Arce, C., \& Gutierrez, S. (1993). Gender differences in the EEG during cognitive activity. International Journal of Neuroscience, 72, 257-264. doi:10.3109/ 00207459309024114

Creed, P. A., Patton, W., \& Bartrum, D. (2002). Multidimensional properties of the LOT-R: Effects of optimism and pessimism on career and wellbeing related variables in adolescents. Journal of Career Assessment, 10, 42-61. doi:10.1177/1069072702010001003

Davidson, R. J. (1985). Affect, cognition, and hemispheric specialization. In C. E. Izard \& J. Kagan (Eds.), Emotions, cognition, and behavior (pp. 320-365). New York: Cambridge University Press.

Davidson, R. J. (1992). Anterior cerebral asymmetry and the nature of emotion. Brain and Cognition, 20, 125-151. doi:10.1016/02782626(92)90065-T

De Pascalis, V., Cozzuto, G., Caprara, G. V., \& Alessandri, G. (2013). Relations among EEG-alpha asymmetry, BIS/BAS, and dispositional optimism. Biological Psychology, 94, 198-209. doi:10.1016/j. biopsycho.2013.05.016

De Pascalis, V., Variale, V., \& D'Antuono, L. (2010). Event-related components of the punishment and reward sensitivity. Clinical Neurophysiology, 121, 60-76. doi:10.1016/j.clinph.2009.10.004
De Raedt, R., Franck, E., Fannes, K., \& Verstraeten, E. (2008). Is the relationship between frontal EEG alpha asymmetry and depression mediated by implicit or explicit self-esteem? Biological Psychology, 77, 89-92. doi:10.1016/j.biopsycho.2007.06.004

Depaoli, S., \& van de Schoot, R. (2017). Improving transparency and replication in Bayesian statistics: The WAMBS-Checklist. Psychological Methods. doi:10.1037/met0000065

Drevets, W. C., Price, J. L., Simpson, J. R., Todd, R. D., Reich, T., Vannier, M., \& Raichle, M. E. (1997). Subgenual prefrontal cortex abnormalities in mood disorders. Nature, 386, 824-827. doi:10. $1038 / 386824 \mathrm{a} 0$

Eid, M., Lischetzke, T., Nussbeck, F. W., \& Trierweiler, L. I. (2003). Separating trait effects from trait-specific method effects in multitrait-multimethod models: A multiple-indicator CT-C(M-1) model. Psychological Methods, 8, 38-60. doi:10.1037/1082-989X. 8.1.38

Fecteau, S., Pascual-Leone, A., Zald, D., Liguori, P., Théoret, H., Boggio, P. S., \& Fregni, F. (2007). Activation of prefrontal cortex by transcranial direct current stimulation reduces appetite for risk during ambiguous decision making. Journal of Neuroscience, 27, 62126218. doi:10.1523/JNEUROSCI.3283-07.2007

Fuchs, M., Kastner, J., Wagner, M., Hawes, S., \& Ebersole, J. S. (2002) A standardized boundary element method volume conductor model. Clinical Neurophysiology, 113, 702-712. doi:10.1016/S13882457(02)00030-5

Gelman, A., Carlin, J. B., Stern, H. S., \& Rubin, D. B. (2004). Bayesian data analysis (2nd ed.). Boca Raton: Chapman \& Hall.

Gratton, G., Coles, M. G. H., \& Donchin, E. (1983). A new method for off-line removal of ocular artifacts. Electroencephalography and Clinical Neurophysiology, 55, 468-484. doi:10.1016/00134694(83)90135-9

Gray, J. A. (1982). The neuropsychology of anxiety: An enquiry into the functions of the septo-hippocampal system. Oxford: Oxford University Press.

Hagemann, D., Naumann, E., Thayer, J. F., \& Bartussek, D. (2002). Does resting EEG asymmetry reflect a trait? An application of latent statetrait theory. Journal of Personality and Social Psychology, 82, 619641. doi:10.1037/0022-3514.82.4.6

Hagemann, D., Naumann, E., \& Thayer, J. F. (2001). The quest for the EEG reference revisited: A glance from brain asymmetry research. Psychophysiology, 38, 847-857. doi:10.1111/1469-8986.3850847

Harmon-Jones, E., \& Allen, J. J. B. (1997). Behavioral activation sensitivity and resting frontal EEG asymmetry: Covariation of putative indicators related to risk of mood disorders. Journal of Abnormal Psychology, 106, 159-163. doi:10.1037/0021-843X.106.1.159

Harmon-Jones, E., \& Sigelman, J. (2001). State anger and prefrontal brain activity: Evidence that insult-related relative left-prefrontal activation is associated with experienced anger and aggression. Journal of Personality and Social Psychology, 80, 797-803. doi:10.1037/ 0022-3514.80.5.797

Harris, A. J. L., \& Hahn, U. (2011). Unrealistic optimism about future life events: A cautionary note. Psychological Review, 118, 135-154. doi: 10.1037/a0020997

Hecht, D. (2013). The neural basis of optimism and pessimism. Experimental Neurobiology, 22, 173-199. doi:10.5607/en.2013. 22.3.173

Henriques, J. B., \& Davidson, R. J. (1991). Left frontal hypoactivation in depression. Journal of Abnormal Psychology, 100, 535-545. doi:10. 1037/0021-843X.100.4.535

Herzberg, P. Y., Glaesmer, H., \& Hoyer, J. (2006). Separating optimism and pessimism: A robust psychometric analysis of the Revised Life Orientation Test (LOT-R). Psychological Assessment, 18, 433-438. doi:10.1037/1040-3590.18.4.433

Jeffreys, H. (1961). The theory of probability (3rd ed.). Oxford: Oxford University Press. 
John, O. P., \& Robins, R. W. (1994). Accuracy and bias in self-perception: Individual differences in self-enhancement and the role of narcissism. Journal of Personality and Social Psychology, 66, 206219. doi:10.1037/0022-3514.66.1.206

Jurcak, V., Tsuzuki, D., \& Dan, I. (2007). 10/20, 10/10, and 10/5 systems revisited: Their validity as relative head-surface-based positioning systems. NeuroImage, 34, 1600-1611. doi:10.1016/j.neuroimage. 2006.09.024

Kahneman, D., \& Tversky, A. (1973). On the psychology of prediction. Psychological Review, 80, 237-251. doi:10.1037/h0034747

Kamarajan, C., Rangaswamy, M., Chorlian, D. B., Manz, N., Tang, Y., Pandey, A. K., ... \& Porjesz, B. (2008). Theta oscillations during the processing of monetary loss and gain: A perspective on gender and impulsivity. Brain Research, 1235, 45-62. doi:10.1016/j.brainres. 2008.06.051

Kingery, L. R. (2003). The psychological correlates of asymmetric cerebral activation (Unpublished dissertation). Retrieved from https:// library.umaine.edu/theses/pdf/KingeryLR2003.pdf

Krain, A., Hefton, S., Pine, D., Ernst, M., Castellanos, F. X., Klein, R., \& Milham, M. (2006). An fMRI examination of developmental differences in the neural correlates of uncertainty and decision-making. Journal of Child Psychology and Psychiatry, 47, 1023-1030. doi: 10.1111/j.1469-7610.2006.01677.x

Kubzansky, L. D., Kubzansky, P. E., \& Maselko, J. (2004). Optimism and pessimism in the context of health: Bipolar opposites or separate constructs? Personality and Social Psychology Bulletin, 30, 943956. doi: $10.1177 / 0146167203262086$

Lindsley, D. B., \& Wicke, J. D. (1974). The electroencephalogram: Autonomous electrical activity in man and animals. In R. Thompson \& M. N. Patterson (Eds.), Bioelectric recording techniques (pp. 3-79). New York: Academic Press.

Maruta, T., Colligan, R. C., Malinchoc, M., \& Offord, K. P. (2000). Optimists vs. pessimist: Survival rate medical patients over a 30 year period. Mayo Clinic Proceedings, 75, 140-143.

Maxwell, J. S., \& Davidson, R. J. (1992). Emotion as motion: Asymmetries in approach and avoidant actions. Psychological Science, 18, 1113-1119. doi:10.1177/0146167208328330

Mazziotta, J., Toga, A., Evans, A., Fox, P., Lancaster, J., Zilles, K., ... \& Mazoyer, B. (2001). A probabilistic atlas and reference system for the human brain: International Consortium for Brain Mapping (ICBM). Philosophical Transactions of the Royal Society B, 356, 1293-1322.

McNeish, D. (2016). On using Bayesian methods to address small sample problems. Structural Equation Modeling, 23, 750-773. doi:10. 1080/10705511.2016.1186549

McPherson, J., \& Mohr, P. (2005). The role of item extremity in the emergence of keying-related factors: An exploration with the Life Orientation Test. Psychological Methods, 10, 120-131. doi:10. 1037/1082-989X.10.1.120

Meharabian, A., \& Ljunggren, E. (1997). Dimensionality and content of optimism-pessimism analyzed in terms of the PAD Temperament Model. Personality and Individual Differences, 23, 729-737. doi: 10.1016/S0191-8869(97)00119-0

Miller, E. K., \& Cohen, J. D. (2001). An integrative theory of prefrontal cortex function. Annual Review of Neurosciences, 24, 167-202. doi: 10.1146/annurev.neuro.24.1.167

Muthén, B., \& Asparouhov, T. (2012). Bayesian SEM: A more flexible representation of substantive theory. Psychological Methods, 17, 313-335. doi:10.1037/a0026802

Muthén, L. K., \& Muthén, B. O. (2012). Mplus user's guide. Los Angeles: Muthén \& Muthén.

Nakamura, K., Honda, M., Hirano, S., Oga, T., Sawamoto, N., Hanakawa, T., ... \& Shibasaki, H. (2002). Modulation of the visual word retrieval system in writing: A functional MRI study on the Japanese orthographies. Journal of Cognitive Neurosciences, 14, 104-115. doi:10.1162/089892902317205366
Pascual-Marqui, R. D., \& Suppl. D. (2002). Standardized low-resolution brain electromagnetic tomography (sLORETA): Technical details. Methods and Findings in Experimental and Clinical Pharmacology, $24,5-12$.

Paulhus, D. L., \& John, O. P. (1998). Egoistic and moralistic biases in self-perception: The interplay of self-deceptive styles with basic traits and motives. Journal of Personality, 66, 1025-1060. doi:10. 1111/1467-6494.00041

Peterson, C. (2000). The future of optimism. American Psychologist, 55, 44-54. doi:10.1037/0003-066X.55.1.44

Peterson, C. K., Gable, P., \& Harmon-Jones, E. (2008). Asymmetrical frontal ERPs, emotion, and behavioral approach/inhibition sensitivity. Social Neuroscience, 3, 113-124. doi:10.1080/ 17470910701612736

Rauch, W. A., Schweizer, K., \& Moosbrugger, H. (2007). Method effects due to social desirability as a parsimonious explanation of the deviation from unidimensionality in LOT-R scores. Personality and Individual Differences, 42, 1597-1607. doi:10.1016/j.paid.2006. 10.035

Reise, S. P., Morizot, J., \& Hays, R. D. (2007). The role of the bifactor model in resolving dimensionality issues in health outcomes measures. Quality of Life Research, 16, 19-31. doi:10.1007/s11136007-9183-7

Robinson-Whelan, S., Kim, C., MacCallum, R. C., \& Kiecolt-Glaser, J. K. (1997). Distinguishing optimism from pessimism in older adults: Is it more important to be optimistic or not to be pessimistic? Journal of Personality and Social Psychology, 73, 1345-1353. doi:10.1037/ 0022-3514.73.6.1345

Rouder, J. N., Speckman, P. L., Sun, D., Morey, R. D., \& Iverson, G. (2009). Bayesian $t$ tests for accepting and rejecting the null hypothesis. Psychonomic Bulletin \& Review, 16, 225-237. doi:10.3758/ PBR.16.2.225

Rubia, K., Lee, F., Cleare, A. J., Tunstall, N., Fu, C. H., Brammer, M., \& McGuire, P. (2005). Tryptophan depletion reduces right inferior prefrontal activation during response inhibition in fast, event-related fMRI. Psychopharmacology, 179, 791-803. doi:10.1007/s00213004-2116-z

Schaffer, C. E., Davidson, R. J., \& Saron, C. (1983). Frontal and parietal electroencephalogram asymmetry in depressed and nondepressed subjects. Biological Psychiatry, 18, 753-762.

Scheier, M. F., \& Carver, C. S. (1985). Optimism, coping, and health: Assessment and implications of generalized outcome expectancies. Health Psychology, 4, 219-247. doi:10.1037/ 0278-6133.4.3.219

Scheier, M. F., \& Carver, C. S. (1992). Effects of optimism on psychological and physical well-being: Theoretical overview and empirical update. Cognitive Therapy and Research, 16, 201-228. doi:10. 1007/BF01173489

Scheier, M. F., \& Carver, C. S. (2001). Adapting to cancer: The importance of hope and purpose. In A. Baum \& B. L. Andersen (Eds.), Psychosocial interventions for cancer (pp. 15-36). Washington, DC: American Psychological Association.

Scheier, M. F., Carver, C. S., \& Bridges, M. W. (1994). Distinguishing optimism from neuroticism (and trait anxiety, self-mastery, and self-esteem): A reevaluation of the Life Orientation Test. Journal of Personality and Social Psychology, 67, 1063-1078. doi:10.1037/0022-3514.67.6. 1063

Scheines, R., Hoijtink, H., \& Boomsma, A. (1998). Bayesian estimation and testing of structural equation models. Psychometrika, 64, 37-52. doi:10.1007/BF02294318

Sharot, T., Kanai, R., Marston, D., Korn, C. W., Rees, G., \& Dolan, R. J. (2012). Selectively altering belief formation in the human brain. Proceeding of the National Academy of Sciences, 109, 1705817062. doi:10.1073/pnas.1205828109 
Sharot, T., Riccardi, M. A., Raio, C. M., \& Phelps, E. A. (2007). Neural mechanisms mediating optimism bias. Nature, 450, 102-105. doi: 10.1038 /nature 06280

Sijtsma, K. (2009). On the use, the misuse, and the very limited usefulness of Cronbach's alpha. Psychometrika, 74, 107-120. doi:10. 1007/s11336-008-9101-0

Somerville, L. H., Kelley, W. M., \& Heatherton, T. F. (2010). Self-esteem modulates medial prefrontal cortical responses to evaluative social feedback. Cerebral Cortex, 20, 3005-3013. doi:10.1093/cercor/ bhq049

Song, X. Y., \& Lee, S. Y. (2012). Basic and advanced Bayesian structural equation modeling: With applications in the medical and behavioral sciences. Chichester: Wiley.

Sutton, S. K., \& Davidson, R. J. (1997). Prefrontal brain asymmetry: A biological substrate of the behavioral approach and inhibition systems. Psychological Science, 8, 204-210. doi:10.1111/j.1467-9280. 1997.tb00413.x

Talairach, J., \& Tournoux, P. (1988). Co-planar stereotaxic atlas of the human brain: 3-dimensional proportional system. An approach to cerebral imaging. Stuttgart: Thieme.

Tamagni, C., Palla, A., Krummenacher, P., Vitacco, D., Huberle, E. Straumann, D., ... \& Brugger, P. (2010). Vestibular stimulation reduces unrealistic optimism. Nature Precedings. Available from http://hdl.handle.net/10101/npre.2010.4519.1

Taylor, S. E., Kemeny, M. E., Reed, G. M., Bower, J. E., \& Gruenewald, T. L. (2000). Psychological resources, positive illusions, and health. American Psychologist, 55, 99-109. doi:10.1037/0003-066X.55.1.99

Tomarken, A. J., Davidson, R. J., Wheeler, R. E., \& Kinney, L. (1992). Psychometric properties of resting anterior EEG asymmetry:
Temporal stability and internal consistency. Psychophysiology, 29, 576-592. doi:10.1111/j.1469-8986.1992.tb02034.x

van Honk, J., Schutter, D. J. L. G., Putman, P., de Haan, E. H. F., \& d'Alfonso, A. A. L. (2003). Reductions in phenomenological, physiological and attentional indices of depressive mood after $2 \mathrm{~Hz}$ rTMS over the right parietal cortex in healthy human subjects. Psychiatry Research, 120, 95-101. doi:10.1016/S0165-1781(03) 00114-8

Vautier, S., Raufaste, E., \& Cariou, M. (2003). Dimensionality of the Revised Life Orientation Test and the status of filler items. International Journal of Psychology, 38, 390-400. doi:10.1080/ 00207590344000222

Vecchione, M., Alessandri, G., Tisak, J., \& Caprara, G. V. (2014). Are method effects permanent or ephemeral in nature? The case of the Revised Life Orientation Test. Structural Equation Modeling, 21, 117-130. doi:10.1080/10705511.2014.859511

Wagner, M., Fuchs, M., \& Kastner, J. (2004). Evaluation of sLORETA in the presence of noise and multiple sources. Brain Topography, 16, 277-280. doi:10.1023/B:BRAT.0000032865.58382.62

Wetzels, R., \& Wagenmakers, E.-J. (2012). A default Bayesian hypothesis test for correlations and partial correlations. Psychonomic Bulletin \& Review, 19, 1057-1064. doi:10.3758/s13423-012-0295-x

Yuan, Y., \& MacKinnon, D. P. (2009). Bayesian mediation analysis. Psychological Methods, 14, 301-322. doi:10.1037/a0016972

Zelazo, P. D., \& Müller, U. (2002). Executive function in typical and atypical development. In U. Goswami (Ed.), Handbook of childhood cognitive development (pp. 445-469). Oxford: Blackwell. 